\title{
Modal analysis of the impact of the boundaries on transverse Anderson localization in a one-dimensional disordered optical lattice
}

\author{
Behnam Abaie ${ }^{\mathrm{a}}$, Seyed Rasoul Hosseini ${ }^{\mathrm{b}}$, Salman Karbasic ${ }^{\mathrm{c}}$, Arash Mafi,* \\ ${ }^{a}$ Department of Physics $\&$ Astronomy and Center for High Technology Materials, \\ University of New Mexico, Albuquerque, NM 87131, USA. \\ ${ }^{b}$ Electrical Engineering Department, University of Wisconsin-Milwaukee, Milwaukee, WI \\ 53201, USA. \\ ${ }^{c}$ Department of Electrical and Computer Engineering, University of California, San \\ Diego, La Jolla, CA 92093, USA.
}

\begin{abstract}
Impact of the boundaries on transversely localized modes of a truncated one-dimensional disordered optical lattice is numerically studied. The results show lower modal number density near the boundaries compared with the bulk, while the average decay rate of the tail of localized modes is the same near the boundaries as in the bulk. It is suggested that the perceived suppressed localization near the boundaries is due to a lower mode density: on average, it is less probable to excite a localized mode near the boundaries; however, once it is excited, its localization is with the same exponential decay rate as any other localized mode.
\end{abstract}

Keywords: Disordered waveguides, Transverse Anderson localization, Boundary effect.

\section{Introduction}

Anderson localization was originally described as the absence of diffusion for electrons in random electronic lattices due to strong scattering and

\footnotetext{
${ }^{\star}$ A. Mafi, S. R. Hosseini, and S. Karbasi acknowledge support by grant number 1029547 from the National Science Foundation.

*Corresponding author

Email address: mafi@unm.edu (Arash Mafi)
}

Preprint submitted to Optics Communications

December 1, 2015

(C) 2015. This manuscript version is made available under the Elsevier user license http://www.elsevier.com/open-access/userlicense/1.0/ 
interference $[1,2]$. However, it was later realized that the concept is inherently a wave phenomenon and was observed in highly scattering classical wave systems including optics, acoustics, elastics, and electromagnetics $[5,4,6,3,7,8,9]$, and various quantum optical systems, such as atomic lattices [10]. Transverse Anderson localization was introduced by Abdullaev, et al. [11] and De Raedt, et al. [12] where a beam of light is localized in transverse dimension(s) of a transversely disordered waveguide but propagates freely in the longitudinally invariant direction. Several observations of transverse Anderson localization have been reported over the past few years $[14,15,13,16,17,18]$.

Recently, Anderson localization of optical waves near the boundaries has been discussed theoretically [20, 19] and experimentally [21, 22]. A delocalizing effect near the boundaries of one dimensional (1D) and two dimensional (2D) random lattice waveguides has been reported, so that a higher level of disorder near the boundaries is claimed to be needed to obtain the same level of localization as in the bulk [20,21]. These reports seemed to be in contrast with our experimental observation of transverse Anderson localization in a glass optical fiber, where a strong localization happens near the outer boundary of the fiber and no trace of localization is observed in the central regions [23]. The disagreements were explained by considering the non-uniform distribution of disorder in the fiber. The disorder was observed to be much stronger near the outer boundary of the fiber which resulted in stronger localization in that region.

In Ref. [21], lower mode density near the boundary of lattice is considered as the reason behind the less localized average intensity profile, which is obtained both experimentally and numerically using the beam propagation method (BPM) [24, 25]. For the BPM, an initial excitation profile is propagated through the lattice waveguide and the final output pattern reveals the extent of localization. To uncover the impact of the boundary on localization, the initial excitation profile is adjusted once to cover the central regions of the waveguide and then its edges, and the output beam profiles are compared with each other. However, this method is not independent of the excitation profile and it is desirable to use a method that can quantify the effect of the boundary on localization independent of the profile of the input beam $[26,27]$.

Recently, Karbasi et al. [28] used a modal analysis to explore localization behavior of a disordered lattice waveguide. The modal analysis offers a clear intuitive description of the localization phenomenon independent of 
the physical properties of the external excitation. Here, we carry out a detailed numerical investigation for the effect of boundaries on the formation of localized modes of a 1D disordered optical lattice waveguide using the modal perspective. Our results show that the average decay rate of the tail of Anderson localized modes is the same near the boundaries as in the bulk. Of course, the boundary-side tail of any mode which is localized near the boundary decays according to the boundary index step; however, the side that faces the lattice decays with the same average exponent as any other mode which is located anywhere else in the lattice.

Another observation presented here is that the modal number density near the boundaries is lower compared with the bulk, confirming similar results obtained by Ref. [21]. Therefore, we suggest that the suppressed localization near the boundaries is due to a lower mode number density rather than a weaker exponential decay of the near-boundary localized modes. In other words, it is less probable to excite a localized mode near the boundaries; however, once it is excited, its localization is with the same exponential decay rate as any other mode (on the disordered lattice side).

\section{Modal Analysis}

Modal analysis as presented in Refs. [14, 29, 30] has two main advantages over other techniques such as the beam propagation method for the investigation of the localization-related phenomena in disordered optical waveguides: 1) A standard technique to analyze optical waveguides is the modal method. The modal method provides an intuitive framework to understand the results related to the transverse Anderson localization of light in a language that is quite familiar in the domain of optical waveguides. 2) The modal analysis allows us to study the localization phenomenon independent of the excitation profile while containing all the information provided by the beam propagation method [28]. In this work, we have chosen to calculate the transverse electric (TE) guided modes of the disordered waveguide using the finite element method (FEM) presented in Ref. [31].

Refractive index profile of the disordered lattice in this article is the same as the one in Ref. [28]. Briefly, the wave-guiding profile is a disordered lattice, which is surrounded by a cladding with refractive index $n_{c}$ on both sides. The disordered lattice is constructed by stacking a collection of dielectric slabs with refractive index of $n_{0}$ and $n_{1}\left(n_{0}<n_{1}\right)$. In our analysis, we have chosen the refractive index of cladding region as $n_{c}=n_{0}$, which 

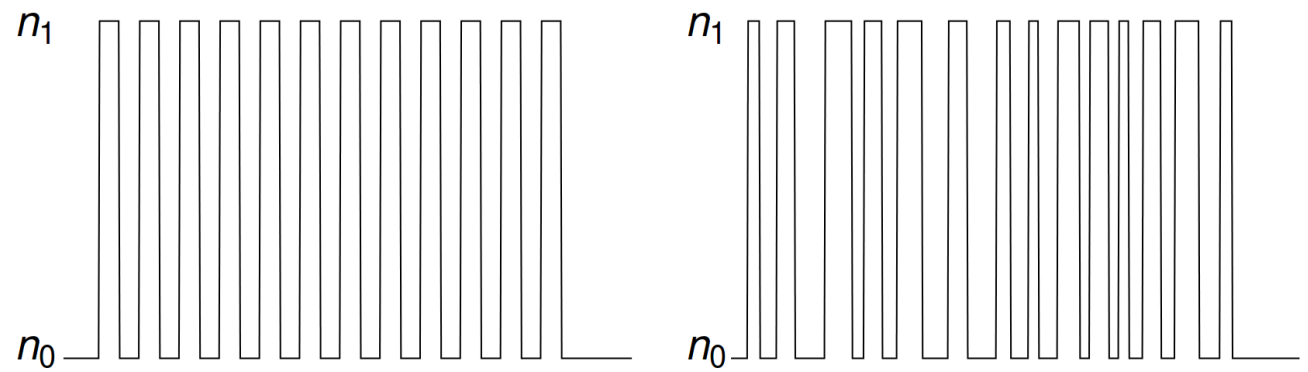

Figure 1: Sample refractive index profiles of ordered (left) and disordered (right) slab waveguides are shown.

resembles the practical disordered waveguides written on the silica glass by femtosecond pulses $[32,21,33]$. Fig. 1(a) shows the refractive index profile of an ordered waveguide versus a disordered one, where for the ordered waveguide the thickness of each slab is chosen to be $\bar{\Lambda}=2 \lambda$ while for the disordered waveguide the thickness of each slab is randomly chosen with uniform probability to be in the interval of $[\bar{\Lambda}-\delta \Lambda, \bar{\Lambda}+\delta \Lambda]$ with $\delta \Lambda=\lambda$. The strength of spatial disorder is defined as $\delta \Lambda / \bar{\Lambda}$, which is kept fixed at $50 \%$ level in this manuscript for simplicity. However, the reported observations are general and apply to other levels of spatial disorder. In order to observe transverse Anderson localization, the disorder can be introduced in many different ways into the waveguide, such as diagonally [1] or off-diagonally [15, 34, 35]; our method is intended to be closely related to the recent implementations of disordered optical fibers of $[12,16]$, and relies on a combination of diagonal and off-diagonal disorder. Fig. 1(b) shows the refractive index profile of the disordered structure.

For each guided mode, using Eqs. 1-4, position (x), width $\left(\sigma_{2}\right)$, asymmetry $\left(\sigma_{3}\right)$, and skewness $(\mathrm{S})$ of the mode across the waveguide [36] are 
calculated, respectively, according to:

$$
\begin{aligned}
\bar{x} & =\int_{-\infty}^{+\infty} x I(x) d x \\
\sigma_{2}^{2} & =\int_{-\infty}^{+\infty}(x-\bar{x})^{2} I(x) d x, \\
\sigma_{3}^{3} & =\int_{-\infty}^{+\infty}(x-\bar{x})^{3} I(x) d x, \\
S & =\frac{2 \sigma_{3}}{\sigma_{2}^{3}} .
\end{aligned}
$$

$I(x)$ is the intensity of the mode in the waveguide and it is normalized such that $\int_{-\infty}^{+\infty} I(x) d x=1$. In these equations, $\bar{x}$ is a measure of the position of the modes across the lattice, $\sigma_{2}$ is a measure of width of the modes meaning that a larger $\sigma_{2}$ is proportional to a wider mode intensity profile distribution, $\sigma_{3}$ is a measure of mode asymmetry in a sense that a zero value of $\sigma_{3}$ is equivalent to a totally symmetric mode, and $S$ is a measure of mode asymmetry, like the third moment $\left(\sigma_{3}\right)$, except that it is normalized by the mode width.

We investigate these characteristic features for 2000 realizations of the random lattice for several values of refractive index difference $(\Delta n)$. To study the localization behavior as a function of location in the waveguide, the lattice is divided into 24 equal bins of width $10 \lambda$ each. The $\mathrm{n}$ th bin on the horizontal line is identified by the position belonging to the interval, $x \in[(10 n+5) \lambda,(10 n+15) \lambda]$, where $n=0,1,2, \ldots, 23 . x=25 \lambda(x=225 \lambda)$ signifies the leftmost (rightmost) side of the disordered lattice, and $x=0$ $(x=250 \lambda)$ signifies the leftmost (rightmost) corner of the cladding in the waveguide.

All the calculated modes are categorized based on their position, where each mode is placed in one of the bins. Each bin is associated with certain number of modes and the statistics associated with that bin can be studied independently. Therefore, we can judge with sufficient statistics whether the position in the waveguide and the distance from the boundaries affect the characteristics (width, spatial density, skewness and decay exponent) of the modes. The simulations are carried out for a wavelength of $\lambda=1 \mu \mathrm{m}, n_{1}=1.5$, $n_{0}=n_{1}-\Delta n$ and the refractive index of cladding $n_{c}$ is equal to $n_{0}$. In this article we consider four cases by varying the refractive index difference $\Delta n$ from 0.0075 to 0.0150 . 


\section{Results and discussion}

In the following, we will discuss the average width, exponential decay rate, asymmetry (skewness), and spatial density of the localized modes. In particular, we are interested in the impact of the boundary on these parameters. The results are presented in several figures, in which, the red filled circles indicate average values, and the error bars show one standard deviation around the mean, calculated over the relevant statistical ensemble.

\subsection{Average width of the localized modes}

The average width of the localized modes can be used as a measure of the localization strength $[28,37]$. Smaller mode widths indicate a stronger localization and vice versa. In this section, we study the average width of the modes across the waveguide in order to investigate whether the position of the modes has an observable effect on their average localized width or not. We observe that the lattice boundary affects the mode width merely by cutting off the intensity profile due to the lattice boundary index step and does not affect the localization strength on the disordered lattice side.

In order to carry out the simulation, we sort the calculated modes based on their positions in the lattice waveguide. In total, 2000 realizations of the lattice waveguide are generated and the modes corresponding to $10 \lambda$ wide intervals are bundled together in bins. The average mode width and the standard deviation are calculated for each bin independently; therefore, values are obtained for each position on the lattice from the corresponding bin.

Fig. 2 shows the average width of the localized modes across the waveguide for various values of refractive index difference $\Delta n$. The horizontal coordinate represents the mean position of the bin on the lattice waveguide. The vertical coordinate corresponds to the width of the modes corresponding to that bin, where each red filled circle represents the average width of the mode bin in that position and the error bar is the standard deviation.

The average width of the localized modes is smaller near the boundaries in comparison with those in the bulk regions. This observation is simply due to the step index interface in the boundary where one side of the intensity profile of the mode is nearly cut off. So, lower mode width does not reflect weaker localization on the disordered lattice side.

The subfigures (a), (b), (c), and (d) in Fig. 2 correspond to $\Delta n=0.0075$, 0.0100, 0.0125, and 0.0150, respectively. By comparing the subfigures, it can 


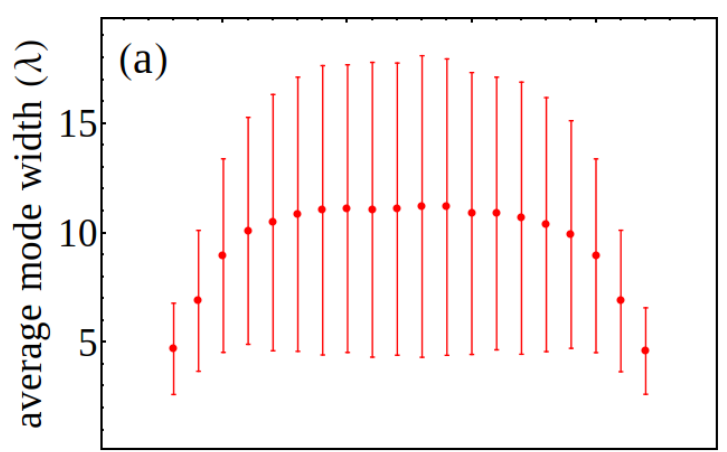

(b)

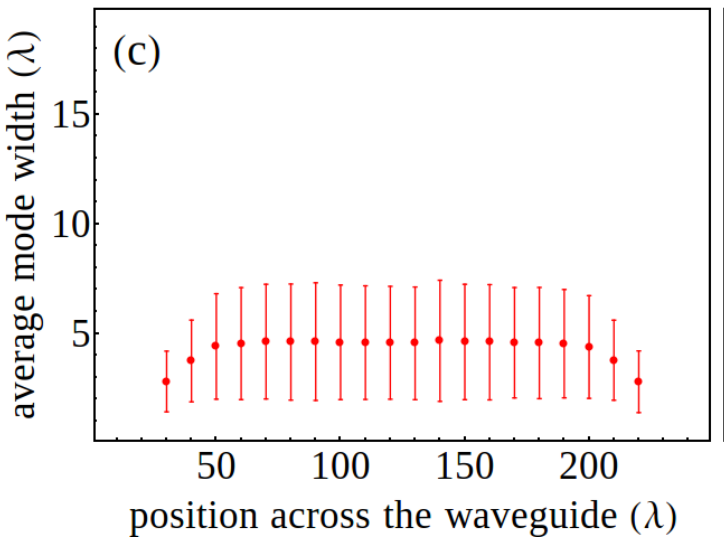

(d)

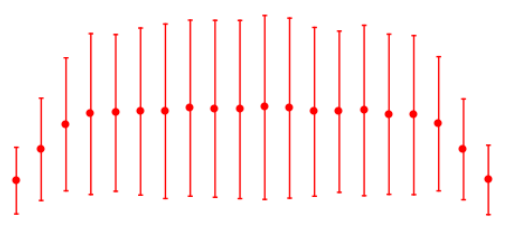

Figure 2: Average width of the modes across the disordered lattice are plotted for (a) $\Delta n$ $=0.0075$, (b) $\Delta n=0.0100$, (c) $\Delta n=0.0125$, and (d) $\Delta n=0.0150$.

be concluded that the disordered lattice refractive index difference $\Delta n$ plays an important role in the localization of light in a disordered lattice [28]. As $\Delta n$ grows, average mode width becomes smaller; therefore, Fig. 2(a) shows the weakest and Fig. 2(d) shows the strongest localization. The modes in Fig. 2(d) are typically confined to only a few slabs. As a result, the boundaries can affect only the modes that are located very close to the boundaries. On the contrary, for Fig. 2(a), the boundary effect propagates all the way through the lattice impacting all the modes throughout the lattice.

In the next section, we will explore the average exponential decay rate of the localized modes and draw conclusions that are consistent with those presented in this section.

\subsection{Average exponential decay rate of the localized modes}

The localization length $L_{c}$ is a commonly used metric to quantify Anderson localization, which is defined through logarithmic average of the lo- 


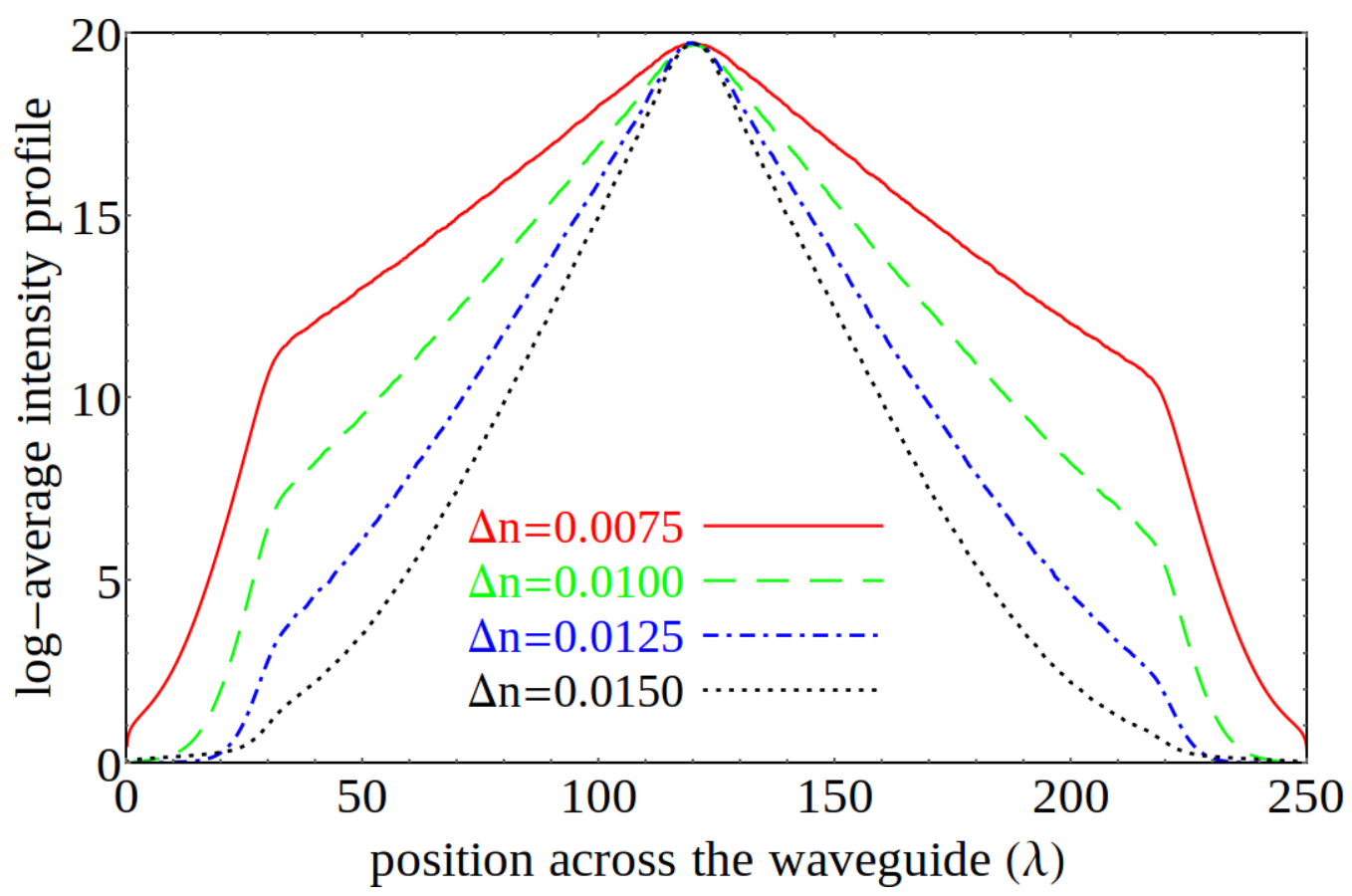

Figure 3: Log-average intensity profile of the modes in a disordered lattice for $\Delta n=$ $0.0075, \Delta n=0.0100, \Delta n=0.0125$, and $\Delta n=0.0150$. Smaller index difference results in a weaker average decay. The profile corresponding to $\Delta n=0.0075$ clearly shows the effect of the lattice-cladding index step.

calized beam profile intensity (modulus-squared) [38]. For a slab waveguide, in the absence of localization effect, the intensity profile of a propagating mode covers the entire lattice width. Of course, the boundary-side tail of the propagating mode decays according to the boundary index step but the intensity profile is spread over the entire lattice. However, in the presence of localization effect, the tail of the intensity profile of a localized mode decays exponentially, for which the decay rate is a measure of localization strength. A higher localization strength results in a faster exponential decay.

In Fig. 3, we plot the logarithmic average of the intensity of the normalized modes of the disordered lattice. After solving for the guided modes of the lattice, we shift the center of each mode defined by Eq. 1 to the center of the lattice. We then repeat this over 2000 statistically identical lattices. We then take the logarithmic average of the mode profile intensity (modulus-squared) of all these center-aligned modes. The resulting log-average intensity profiles are plotted for $\Delta n=0.0075, \Delta n=0.0100, \Delta n=0.0125$, and $\Delta n=0.0150$. 
As expected, the log-average intensity in Fig. 3 shows an exponential decay behavior as $\exp \left(-|x| / L_{c}\right)$. The localization length is clearly smaller for larger values of disorder. For the case with $\Delta n=0.0150$, the decay is so strong that-on average-the tails of the modes that are near the center of the lattice are hardly affected by the boundary. In contrast, for $\Delta n=0.0075$, the disorder-induced decay rate is small and the exponentially decaying tail of the modes is eventually terminated at the boundary by the lattice-cladding index step. The disorder induced decay exponent is visibly different from the decay exponent due to the lattice-cladding index step in Fig. 3. For the cases of $\Delta n=0.0075$ and $\Delta n=0.0100$, the index contrast is clearly not large enough to create strong localization as observed for $\Delta n=0.0125$ and $\Delta n=0.0150$.

It is clear that the lattice-cladding index step affects the intensity profile of the beam on the side that faces the boundary; the non-trivial question is whether it affects the average exponential decay rate of the opposite side that faces the lattice or not. In other words, we would like to know whether the impact of the boundary is only limited to terminating the tail of a profile and changing its local exponential decay rate, or it actually affects the disorderbased decay rate on the lattice-side of the beam as well. In order to address this question, we compare localization strength for the modes that reside in the central region of the lattice with that of the modes that are near the boundary. The decay rate for the modes near the boundary is calculated from the side of the mode that is facing the disordered lattice (away from the cladding).

Comparisons are made for various values of $\Delta n$ and are reported in Table 1 , where the decay rates are categorized for the modes that are located near the left boundary, at the central bin, and near the right boundary. For each value of $\Delta n$, the average decay exponent is independent of the location of the beam in the lattice; therefore, the boundary does not affect the localization strength on the lattice side of the modes. However, the decay rates are affected by the value of $\Delta n$, as expected. The results presented here are consistent with our observations in the previous section on the impact of the boundary on the average mode width.

\subsection{Asymmetry of the localized modes}

From the results in the previous section, we learned that the effect of the boundary is limited to the modes that reside very close to the boundary. The impact of the boundary is only in changing the exponential decay rate of a 
Table 1: Average exponential decay exponent of the localized modes in units of $\lambda^{-1}$ are reported at the leftmost, central, and rightmost bins of the lattice for different values of $\Delta n$. The decay rates of the near-boundary modes are obtained from the tail of the modes that are on the side of the lattice.

\begin{tabular}{cccc}
\hline$\Delta n$ & leftmost bin & central bin & rightmost bin \\
\hline$\Delta n=0.0075$ & 0.0998 & 0.0962 & 0.0988 \\
$\Delta n=0.0100$ & 0.1432 & 0.1432 & 0.1418 \\
$\Delta n=0.0125$ & 0.1864 & 0.1862 & 0.1848 \\
$\Delta n=0.0150$ & 0.2036 & 0.2060 & 0.2020 \\
\hline
\end{tabular}

mode on the cladding side of the lattice, and the lattice side of the mode is not affected (on average). Therefore, the modes are skewed asymmetrically near the boundary.

The third central moment from Eq. 3 can be used as a measure of the asymmetry of the modes, where zero value for third moment denotes a symmetric mode. However, $\sigma_{3}$ can be misleading unless it is properly normalized by the mode width. The more appropriate measure is the skewness from Eq. 4, which is a relative metric that takes into account the impact of mode width.

Fig. 4 shows the average values of calculated skewness of the localized modes across the waveguide for various values of $\Delta n$. For all values of $\Delta n$, calculated skewness is almost zero in the central regions, where the modes are left-right symmetric on the average. However, it is non-zero near the boundaries, because the tails of the modes are nearly truncated at the boundaries due to the lattice-cladding index step. The truncation is strongest for the case of $\Delta n=0.0150$, as expected, and shown in Fig. $4 \mathrm{~d}$.

\subsection{Spatial density of the localized modes}

A higher mode density means that, on average, it is more probable to excite a localized mode rather than an extended radiation mode; therefore the initial excitation is more likely to remain localized inside the lattice and the localized propagating power is nearly the same as the in-coupling power. Higher mode density is beneficial, for example for image transport through disordered waveguides, where the image fidelity is negatively affected if there are not a sufficiently large number of highly localized modes that can carry the local details of an image [39]. The calculation of the average mode density at each individual bin across the lattice provides an estimate of the probability of localization of an initial excitation in that bin, relative to the 

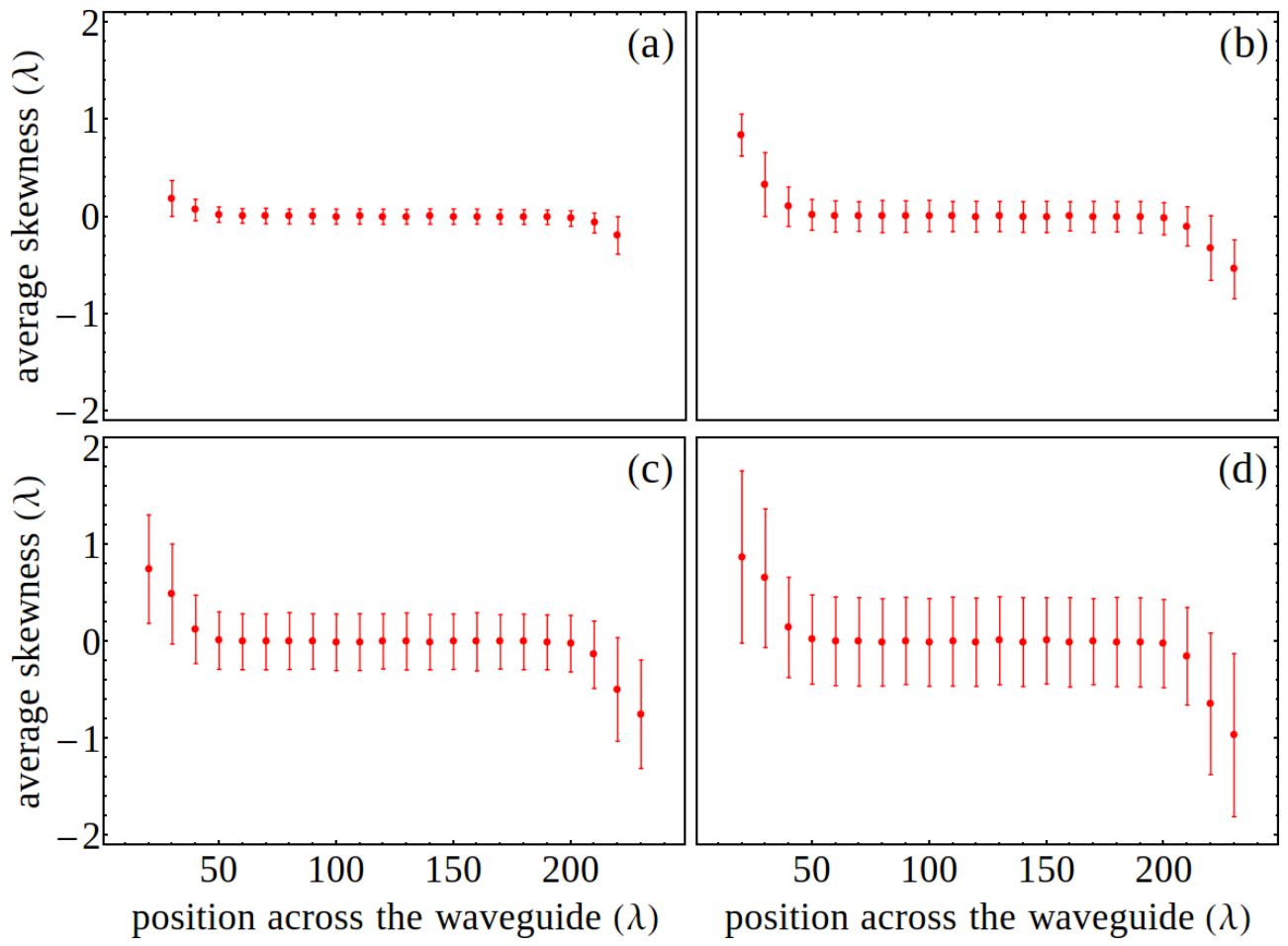

Figure 4: Average skewness of the modes across the disordered lattice are plotted for (a) $\Delta n=0.0075$, (b) $\Delta n=0.0100$, (c) $\Delta n=0.0125$, and (d) $\Delta n=0.0150$.

amount of power that is coupled to the radiation modes. In this section, we calculate the average number of localized modes in each bin across the lattice for $\Delta n=0.0075, \Delta n=0.0100, \Delta n=0.0125$, and $\Delta n=0.0150$, respectively.

Figure 5 shows the spatial density of modes across the disordered lattice for various values of the refractive index difference $\Delta n$. The vertical axis shows the average number of modes in each bin across the lattice. The results indicate a lower number of localized modes near the boundaries compared with the bulk regions. This observation is in accordance with the previous studies in Ref. [21]. As a consequence of lower mode density, on average, it is less probable to excite a localized mode near the boundaries, and in this limited scope, the boundaries have a de-localizing effect.

Another notable feature is the dependency of the average number of modes on the index difference $\Delta n$. For disordered waveguides with higher values of refractive index difference $\Delta n$, the average value of mode density is higher in comparison with those of lower refractive index difference val- 
ues. This behavior is expected, because an increase in the index step $\Delta n$ increases the effective numerical aperture of the waveguiding lattice; therefore, the total number of the modes increases similar to conventional waveguides. We also note that increasing the refractive index difference $\Delta n$ results in a stronger transverse scattering and a weaker coupling between adjacent waveguides. Therefore, the quality of transverse localization is improved for a larger $\Delta n$. This effect also leads to an increase in the number of localized modes.
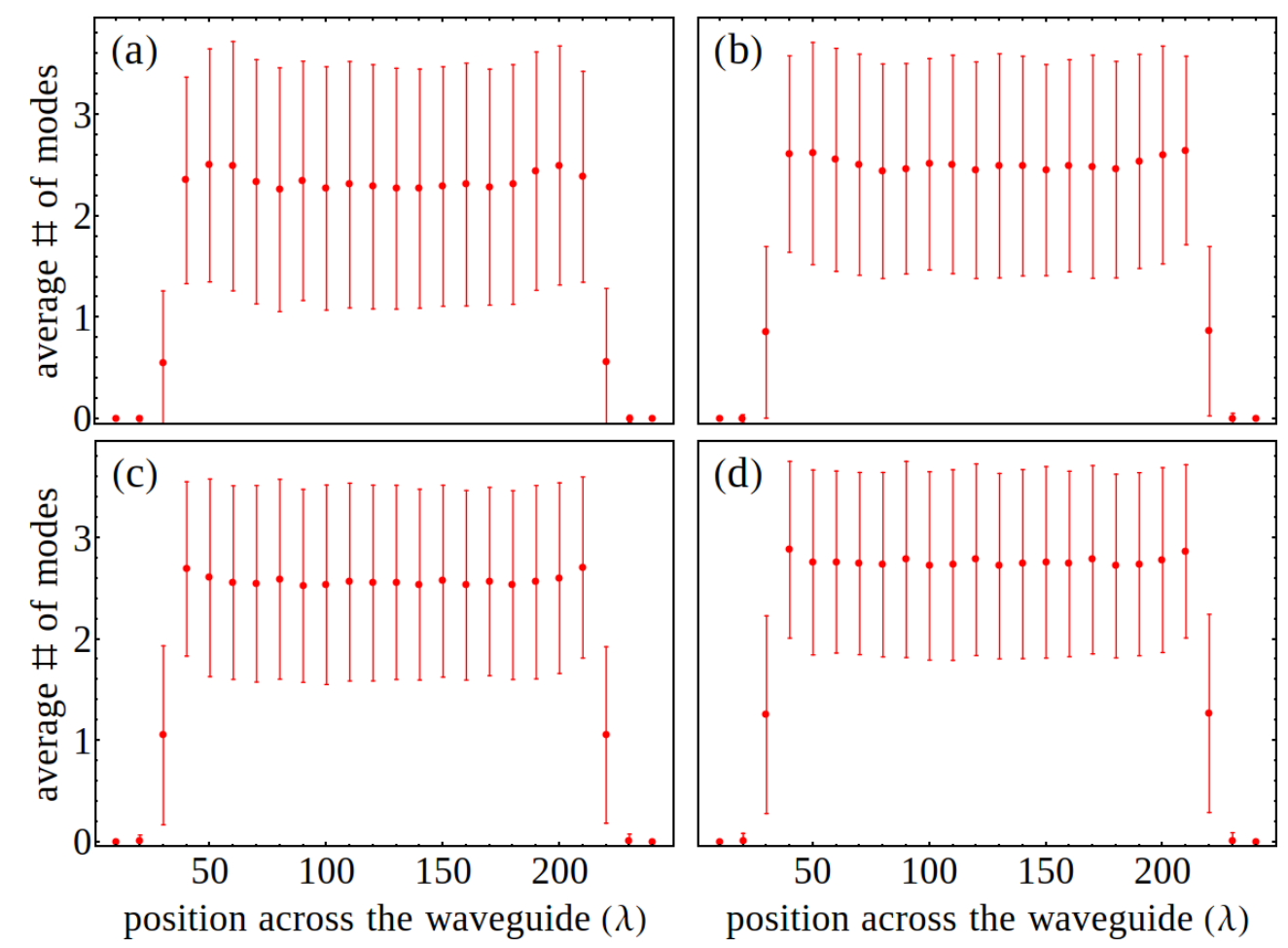

Figure 5: Average density of the modes across the disordered lattice. Subfigures (a), (b), (c), and (d) represent the average density of the modes for $\Delta n=0.0075, \Delta n=0.0100$, $\Delta n=0.0125$, and $\Delta n=0.0150$ respectively.

\section{Conclusion}

We have investigated the impact of the boundaries on important mode parameters of a one dimensional disordered optical lattice using the modal technique. Results show smaller average mode widths near the boundaries, 
but the effect is merely because of the near-truncation effect of the claddingwaveguide index step. For any mode that is localized near the boundary of the lattice, the average decay exponent of the tail on the lattice side is not affected by the boundary and is the same as the decay exponent of the modes that are localized near the center of the disordered lattice waveguide.

The density of the localized modes is lower near the boundaries, as was previously pointed out in Ref. [21]. Therefore, near the boundary, it is less probable to excite a localized mode in comparison with the central regions and more power is coupled to delocalized radiation modes. For image transport experiments such as those in Ref. [39], the transported images can be washed out or pixelated near the boundaries if there are not enough modes to transport the fine details of the image.

In summary, the only non-trivial impact of the boundary is that it lowers the density of the localized modes near the boundary; and only in this limited scope, do the boundaries have a de-localizing effect.

\section{References}

[1] P. W. Anderson, "Absence of diffusion in certain random lattices," Phys. Rev. 109, 1492-1505 (1958).

[2] E. Abrahams, 50 years of Anderson localization (World Scientific, 2010).

[3] P. W. Anderson, "The question of classical localization: A theory of white paint?," Philos. Mag. B 52, 505-509 (1985).

[4] S. John, "Strong localization of photons in certain disordered dielectric superlattices," Phys. Rev. Lett. 58, 2486-2489 (1987).

[5] R. L. Weaver, "Anderson localization of ultrasound," Wave Motion 12, 129-142 (1990).

[6] R. Dalichaouch, J. P. Armstrong, S. Schultz, P. M. Platzman, and S. L. McCall, "Microwave localization by two-dimensional random scattering," Nature 354, 53-55 (1991).

[7] S. John, "Localization of light," Phys. Today 44, 32-40 (1991).

[8] A. A. Chabanov, M. Stoytchev, and A. Z. Genack, "Statistical signatures of photon localization," Nature 404, 850-853 (2000). 
[9] A. D. Lagendijk, B. V. Tiggelen, B., D. S. Wiersma, "Fifty years of Anderson localization," Phys. Today 62, 24-29 (2009).

[10] J. Billy, V. Josse, Z. Zuo, A. Bernard, B. Hambrecht, P. Lugan, D. Clément, L. Sanchez-Palencia, P. Bouyer, and A. Aspect, "Direct observation of Anderson localization of matter waves in a controlled disorder," Nature 453, 891-894 (2008).

[11] S. S. Abdullaev, and F. K. Abdullaev, "On propagation of light in fiber bundles with random parameters," Radiofizika 23, 766-767 (1980).

[12] H. de Raedt, A. D. Lagendijk, and P. de Vries, "Transverse localization of light," Phys. Rev. Lett. 62, 47-50 (1989).

[13] T. Schwartz, G. Bartal, S. Fishman, and M. Segev, "Transport and Anderson localization in disordered two-dimensional photonic lattices," Nature 446, 52-55 (2007).

[14] Y. Lahini, A. Avidan, F. Pozzi, M. Sorel, R. Morandotti, D. N. Christodoulides, and Y. Silberberget, "Anderson localization and nonlinearity in one-dimensional disordered photonic lattices," Phys. Rev. Lett. 100, 013906 (2008).

[15] L. Martin, G. D. Giuseppe, A. Perez-Leija, R. Keil, F. Dreisow, M. Heinrich, S. Nolte, A. Szameit, A. F. Abouraddy, D. N. Christodoulides, and B. E. A. Saleh, "Anderson localization in optical waveguide arrays with off-diagonal coupling disorder," Opt. Express 19, 13636-13646 (2011).

[16] S. Karbasi, C. R. Mirr, P. G. Yarandi, R. J. Frazier, K. W. Koch, and A. Mafi, "Observation of transverse Anderson localization in an optical fiber," Opt. Lett. 37, 2304-2306 (2012).

[17] S. Karbasi, R. J. Frazier, C. R. Mirr, K. W. Koch, and A. Mafi, "Fabrication and characterization of disordered polymer optical fibers for transverse Anderson localization of light," J. Vis. Exp. 77 (2013).

[18] S. Karbasi, K. W. Koch, and A. Mafi, "Multiple-beam propagation in an Anderson localized optical fiber," Opt. Express 21, 305-313 (2013).

[19] M. I. Molina, "Boundary-induced Anderson localization in photonic lattices," Phys. Rev. A 375, 2056-2058 (2011). 
[20] D. M. Jovic, Y. S. Kivshar, C. Denz, and M. R. Belic, "Anderson localization of light near boundaries of disordered photonic lattices," Phys. Rev. A 83, 033813 (2011).

[21] A. Szameit, Y. V. Kartashov, P. Zeil, F. Dreisow, M. Heinrich, R. Keil, S. Nolte, A. Tunnermann, V. A. Vysloukh, L. Torner, "Wave localization at the boundary of disordered photonic lattices," Opt. Lett. 35, 11721174 (2010).

[22] U. Naether, J. M. Meyer, S. Sttzer, A. Tnnermann, S. Nolte, M. I. Molina, A. Szameit, "Anderson localization in a periodic photonic lattice with a disordered boundary," Opt. Lett. 37, 485-487 (2012).

[23] S. Karbasi, T. Hawkins, J. Ballato, K. W. Koch, and A. Mafi, "Transverse Anderson localization in a disordered glass optical fiber," Opt. Mater. Express 2, 1496-1503 (2012).

[24] W. P. Huang, and C. L. Xu, "Simulation of three-dimesional optical waveguides by full-vector beam propagation method," J. Lightwave Technol. 29 2639-2649 (1993).

[25] S. Karbasi, C. R. Mirr, P. G. Yarandi, R. J. Frazier, K. W. Koch, and A. Mafi, "Detailed investigation of the impact of the fiber design parameters on the transverse Anderson localization of light in disordered optical fibers," Opt. Express 20, 18692-18706 (2012).

[26] S. Ghosh, G. P. Agrawal, B. P. Pal, and R. K. Varshney, "Localization of light in evanescently coupled disordered waveguide lattices: Dependence on the input beam profile," Opt. Commun. 284, 201-206 (2011).

[27] S. Ghosh, B. P. Pal, R. K. Varshney, and G. P. Agrawal, "Transverse localization of light and its dependence on the phase-front curvature of the input beam in a disordered optical waveguide lattice," J. Opt. (IOP) 14, 075701:1-5 (2012).

[28] S. Karbasi, K. W. Koch, and A. Mafi, "Modal perspective on the transverse Anderson localization of light in disordered optical lattices," J. Opt. Soc. Am. B 30, 1452-1461 (2013). 
[29] R. G. S. El-Dardiry, S. Faez, and Ad. Lagendijk, "Snapshots of Anderson localization beyond the ensemble average," Phys. Rev. B 86, 125132 (2012).

[30] Y. V. Kartashov, V. V. Konotop, V. A. Vysloukh, and L. Torner, "Light localization in nonuniformly randomized lattices," Opt. Lett. 37, 286288 (2012).

[31] T. A. Lenahan, "Calculation of modes in an optical fiber using the finite element method and EISPACK," Bell Syst. Tech. J. 62, 2663-2694 (1983).

[32] A. Szameit, I. L. Garanovich, M. Heinrich, A. A. Sukhorukov, F. Dreisow, T. Pertsch, S. Nolte, A. Tnnermann, S. Longhi, and Y. S. Kivshar, "Observation of two-dimensional dynamic localization of light," Phys. Rev. Lett. 104, 223903 (2010).

[33] S. Ghosh, N. D. Psaila, R. R. Thomson, B. P. Pal, R. K. Varshney, and A. K. Kar, "Ultrafast laser inscribed waveguide lattice in glass for direct observation of transverse localization of light," Appl. Phys. Lett. 100, 101102 (2012).

[34] J. B. Pendry, "Off-diagonal disorder and 1D localization," J. Phys. C: Solid State Phys. 15, 5773-5778 (1982).

[35] C. M. Soukoulis, and E. N. Economou, "Off-diagonal disorder in onedimensional systems," Phys. Rev. B 24, 5698-5702 (1981).

[36] A. Mafi and J. V. Moloney, "Beam quality of photonic-crystal fibers," J. Lightwave Technol. 23, 2267- (2005)

[37] M. I. Stockman, S. V. Faleev, and D. J. Bergman, "Localization versus delocalization of surface plasmons in nanosystems: can one state have both characteristics?," Phys. Rev. Lett. 87, 167401-1-4 (2001).

[38] C. M. Soukoulis and E. N. Economou, "Electronic localization in disordered systems," Waves in Random Media 9, 255-269 (1999).

[39] S. Karbasi, R. J. Frazier, K. W. Koch, T. Hawkins, J. Ballato, and A. Mafi. "Image transport through a disordered optical fibre mediated by transverse Anderson localization," Nature Communications 5, 3362 (2014). 